\title{
Short-Term In-Home Intervention Reduces Depressive Symptoms in Early Head Start Latina Mothers of Infants and Toddlers
}

\author{
Linda S. Beeber ${ }^{1,{ }^{\star}}$, Diane Holditch-Davis ${ }^{2,{ }^{* *}}$, Krista Perreira $^{3, \dagger}$, Todd A. Schwartz ${ }^{4, \ddagger}$, \\ Virginia Lewis ${ }^{1, \S}$, Hjordis Blanchard ${ }^{1, \pi}$, Regina Canuso ${ }^{1, \|}$, and Barbara Davis Goldman \\ ${ }^{1}$ School of Nursing, University of North Carolina at Chapel Hill, Chapel Hill, NC \\ ${ }^{2}$ School of Nursing, Duke University, Durham, NC \\ ${ }^{3}$ Department of Public Policy, University of North Carolina at Chapel Hill, Chapel Hill, NC \\ ${ }^{4}$ Department of Biostatistics, Gillings School of Global Public Health and School of Nursing, \\ University of North Carolina at Chapel Hill, Chapel Hill, NC \\ ${ }^{5}$ Frank Porter Graham Child Development Institute, University of North Carolina at Chapel Hill, \\ Chapel Hill, NC
}

\section{Abstract}

Depressive symptoms may compromise the ability of low-income Latina mothers with limited English language proficiency to parent their infants or toddlers. Eighty Early Head Start Latina mothers with limited English language proficiency were randomized to an advanced practice nurse-delivered, culturally tailored, in-home psychotherapy intervention, or to usual care. Repeated measures regression analysis showed a significantly greater decrease in depressive symptoms for intervention mothers compared to the usual care group at 22 and 26 weeks (4 weeks post intervention). Intervention mothers' reports of their child's aggression diminished significantly from $\mathrm{T} 1$ to $\mathrm{T} 4$ compared to usual care mothers $(p=.03)$. Self-efficacy appeared to only partially mediate the intervention effect, and maternal health moderated the intervention impact. Results indicate that the intervention reduced depressive symptoms and, compared to previous studies in this population, retention of mothers in both intervention and control conditions was improved.

\footnotetext{
(c) 2009 Wiley Periodicals, Inc.

Correspondence to Linda S. Beeber, School of Nursing, University of North Carolina at Chapel Hill, CB 7460, Chapel Hill, NC $27599-7460$.

Frances Hill Fox Term Professor.

*** Marcus Hobbs Professor of Nursing and Associate Dean for Research Affairs.

$\dagger$ Associate Professor.

†Research Assistant Professor.

\$Project Manager.

IIData Manager.

Instructor.

${ }^{\#}$ Scientist and Coordinator of Infant Learning and Assessment.
} 


\section{Keywords}

care of minority groups/patients; depression; emotional states/feelings; parenting; parent-infant health/parenting; growth and development; child; developmental stages/events; growth and development; infant; developmental stages/events; parent-child relationships; family health; mental health; health promotion/wellness behaviors

Low-income, Spanish-speaking Latina mothers of infants and toddlers are confronted with chronic stressors: economic hardship, loss of extended family and community support, limited English language proficiency (Flores, Abreu, \& Tomany-Korman, 2005), and pressure to rapidly acculturate into an unfamiliar culture (Chaudron et al., 2005). As many as $64 \%$ of these mothers experience clinically significant depressive symptoms lasting a year or more (Alderete, Vega, Kolody, \& Aguilar-Gaxiola, 2000; Finch, Kolody, \& Vega, 2000; Riolo, Nguyen, Greden, \& King, 2005), which limit their ability to talk, play, comfort, and offer appropriate developmental support to their US-born infant or toddler (Coyl, Roggman, \& Newland, 2002; Forman et al., 2007; Zeanah, Boris, \& Larrieu, 1997).

Exposure to maternal depressive symptoms has been linked to early language deficits, behavioral dysregulation, persistent developmental lags, poor health and healthcare, and in older children, conduct disorders and social aggression (Flores, Abreu, \& Tomany-Korman, 2006; Flores et al., 2002; Flores \& Vega, 1998). Having a mother who is symptomatic for 6 months or longer (Field, 1998) vastly increases these risks (Black, Papas, Hussey, Dubowitz et al., 2002; Breznitz \& Sherman, 1987; Flores et al., 2005). Thus, as the children of these Latina mothers enter the US educational system, they are liable to have less than optimal academic achievement and may need remedial services.

In comparison with other minorities, newly immigrated Latinas have reported high receptivity to receiving treatment (Nadeem et al., 2007), however, factors such as stigma, rural isolation, and lack of access to insurance and transportation create barriers. When Latinos immigrate to new destination areas of the US where there is no established infrastructure of accessible bilingual mental health services, limited English proficiency poses an additional barrier to treatment (Alpert, 2005; Marrow, 2005; Vega, Kolody, Aguilar-Gaxiola, \& Catalano, 1999). The purpose of this study, conducted in a new destination area, was to test a nurse-delivered intervention that could reach symptomatic Latina mothers with these characteristics in a non-stigmatic fashion to help them reduce depressive symptoms and increase their capacity to be responsive to and supportive of their infants and toddlers.

Depressive symptoms can be debilitating due to the unremitting sad mood and changes in cognitive, affective, behavioral, and somatic functioning. Over time, depressive symptoms may progress to more severe mood disorders such as Major Depressive Episodes (Gotlib, Lewinsohn, \& Seeley, 1995; Kessler et al., 2003; Sherbourne et al., 1994). Depressive symptoms compromise low-income, newly immigrated Latina mothers' abilities to learn English, become educated, work, maintain their families' well-being, and support the optimal development of their US-born infants or toddlers (Coyl et al., 2002; Forman et al., 2007; Zeanah et al., 1997). Even at moderate levels, depressive symptoms rob mothers of 
the energy needed to provide developmentally stimulating parenting (Gross, 1989; Hammen, 1991) by limiting the frequency and length of their interactions with the child and slowing responsiveness to the child's signals (Murray, Fiori-Cowley, Hooper, \& Cooper, 1996; Zeanah et al.). Lowered responsiveness impairs the mother's ability to soothe the infant (Zuckerman, Bauchner, Parker, \& Cabral, 1990) and assist the toddler in regulating behavior (Goodman, Brogan, Lynch, \& Fielding, 1993; Needlman, Stevenson, \& Zuckerman, 1991). In addition, symptomatic mothers may interact irritably and erratically with their infants and have difficulty expressing praise for the good behavior of their toddlers (Cohn, Matias, Tronick, Connell, \& Lyons-Ruth, 1986; Cohn \& Tronick, 1989; Rosenblum, Mazet, \& Bénony, 1997). These parenting patterns inhibit the social and emotional maturation of the child (Field, Healy, Goldstein, \& Gutherz, 1990).

Depressive symptoms also have been associated with persistent negative maternal perceptions of the child in the form of unrealistic expectations and critical judgments of the child's behavior (Forman et al., 2007; Kochanska, Radke-Yarrow, Kuczynski, \& Friedman, 1987; Radke-Yarrow, Belmont, Nottelmann, \& Bottomly, 1990). Symptomatic mothers with negative perceptions report more aggression and problem behaviors in their children (Hammen, 1991), express more negative affect toward them (Radke-Yarrow, Nottelmann, Belmont, \& Welsh, 1993), and demonstrate harsh parenting styles (Boyd, Zayas, \& McKee, 2006; Ruttenberg, Finello, \& Cordeiro, 1997). Frequently, these patterns of interacting are accompanied by low parenting self-efficacy (Gross, 1989). Doubly isolated by limited English proficiency and depressive symptoms, symptomatic Latina mothers may be the only adults consistently providing physical care for and interacting with their infants and toddlers. This combination of factors intensifies the negative impact of maternal depressive symptoms during the period of the child's most rapid neurological and psychosocial development. Infants and toddlers exposed to maternal depressive symptoms for 6 months or longer demonstrate profound language delays, and cognitive and developmental deficits (Black, Papas, Hussey, Dubowitz et al., 2002; Black, Papas, Hussey, Hunter et al., 2002; Field, 1998), and as school-aged children, experience behavior problems (A. Jackson, BrooksGunn, Huang, \& Glassman, 2000), learning difficulties, and conduct disorders (Beardslee \& Gladstone, 2001; Brennan, Hammen, Katz, \& Le Brocque, 2002; Lovejoy, Graczyk, O'Hare, \& Neuman, 2000).

To our knowledge, prior to this study only one clinic-based interpersonal intervention had been tested with low-income Latina mothers (Spinelli \& Endicott, 2003). Although the treatment showed effectiveness, attrition was high (32\%). To reduce attrition, several investigators have adapted psychotherapy for in-home delivery to high-risk populations. These trials were conducted with European mothers and were directed exclusively toward the immediate postpartum period (Appleby, Warner, Whitton, \& Faragher, 1997; Chabrol et al., 2002; Wickberg \& Hwang, 1996). Only one included low-income mothers, and none had been adapted for Latina mothers with limited English proficiency. Although one in-home intervention using six psychotherapy sessions and antidepressants showed effectiveness, attrition was still high, with $38 \%$ of the mothers in the intervention condition dropping out prior to completing the trial (Appleby et al.). Interpersonal Therapy (IPT; Weissman, Markowitz, \& Klerman, 2000), a well tested, effective treatment for depressive symptoms, has been modified for use with different populations, including Latino adolescents (Miranda 
et al., 2005) and postpartum women (Stuart \& O'Hara, 1995; Stuart, O'Hara, \& Gorman, 2003), and recently it was adapted for in-home delivery with low-income mothers by the team conducting the current study (Beeber, Holditch-Davis, Belyea, Funk, \& Canuso, 2004). However, the in-home adaptation of IPT has never been tested with Latina mothers with limited English proficiency.

The primary aim of this study was to test the efficacy of a 5-month, in-home, culturally appropriate adaptation of IPT on reducing depressive symptoms in symptomatic low-income Latina mothers. We hypothesized that

1. Mothers who received the intervention would demonstrate significantly less depressive symptom severity, report less child behavioral aggression and fewer concerns about child social-emotional functioning, and demonstrate greater maternal responsiveness midway through the intervention (T2: 14 weeks), at the conclusion of the intervention (T3: 22 weeks), and 1 month following completion of the intervention (T4: 26 weeks) compared to mothers who received usual care.

2. Maternal self-efficacy would mediate the effects of the intervention on depressive symptom severity.

3. Characteristics of the mother (age, education) and maternal burdens (partnered status, number of children in the household under the age of 5 years, acculturation level, maternal health, dependence on others for transportation, child health problems, child use of disability services, legal problems, and level of family conflict) would moderate the effects of the intervention on depressive symptom severity.

\section{METHODS}

\section{Setting and Sample}

The sample was newly immigrated, Latina mothers with depressive symptoms who had infants or toddlers enrolled in three Early Head Start (EHS) programs in a new destination area of the southeastern US, where a 400\% increase in the Hispanic population occurred between 1990 and 2000 (Miller, 2003). Sample size was selected to be able to detect a difference of two-thirds of a standard deviation between the intervention and control groups over the four data collection points (Cohen, 1977; Keyes \& Muller, 1991) and with maximum and minimum correlation between time points of .60 and .45 , respectively. Assuming use of Wilks' Lambda test for the design and an a of .05, we calculated that a total sample size of 72 would adequately detect a time trend difference between the intervention and control group with a power of .80. To compensate for possible attrition, we planned to recruit a total sample of 80 mothers.

Figure 1 presents a Consolidated Standards of Reporting Trials (CONSORT) diagram (Moher, Schulz, \& Altman, 2005) of the study leading to the final sample of 80 EHS Latina mothers. The average Center for Epidemiological Studies Depression scale (CES-D) score at baseline was $24.5(S D=13.0)$, with some mothers scoring as high as 52 (the maximum possible on the CES-D is 60$)$. Sixty-four (80\%) of the mothers completed at least three data 
collections (33 intervention/31 usual care), and two-thirds ( $n=53$ ) completed all four (28 intervention/25 usual care), with nine mothers dropping out after the first data collection (5 intervention/4 usual care). The most frequent reason for withdrawal from the study was the mother moving to a different state or leaving the US (five mothers). Four mothers withdrew from EHS, and the remainder were "too busy" to participate. Thirty-four mothers completed the intervention (87\%), representing an improvement in retention compared to previous studies with low-income mothers and Latina mothers (Appleby et al., 1997; Spinelli \& Endicott, 2003).

The mothers spoke only Spanish or were limited in English language proficiency, indicated by their score on a standardized instrument (see Instruments; SASH). Most (92\%) were born in Mexico, were newly immigrated (mean 5.3 years in US, $S D=3.5$ ) and young (mean $=26$ years, $S D=5.9$ ). Mothers had a range of $2-16$ years of formal education (mean $=8.7$ years, $S D=2.9$ years), $70 \%$ had fewer than 10 years of education, and $30 \%$ had 6 or fewer years. Most were living with a partner (84\%) with as many as nine people in the home; $34 \%$ were working full-time or part-time away from home; $40 \%$ did not drive or depended on others for transportation. The average age of the EHS child was 16 months $(S D=12) ; 20 \%$ of the children had chronic health problems, and $19 \%$ had been referred to services for language or developmental delays or conduct problems. Table 1 summarizes the demographic characteristics.

EHS is a federal child enrichment program providing center-based and home visiting services for low-income infants and toddlers (0-3 years of age). The participating EHS programs enrolled the US-born Latino children of parents who worked in meat processing, construction, landscaping, farming, and service industries. Two of the EHS programs were located in a rural mountainous area and the third was in an urban-suburban area.

The intervention was delivered in partnership with bilingual EHS staff who had gained the trust of symptomatic mothers. Staff were taught to offer every enrolled Latina mother with limited English proficiency the opportunity to participate in the screening phase of the project. To be eligible for screening, in addition to having limited English proficiency, mothers had to be at least 15 years old, the biological mother and primary caretaker of an EHS-enrolled child between 6 weeks and 3 years of age, and able to give consent or assent. Mothers who were younger than 18 years old were required to have a guardian's consent and to sign an assent statement. Mothers were not screened earlier than 6 weeks postpartum in order to allow the resolution of transient depressive symptoms that are common after childbirth (American Psychiatric Association, 2000). After consenting, mothers answered a brief demographic questionnaire and were screened for depressive symptoms with the 20item CES-D (Radloff, 1977), Spanish version (Vega, Kolody, Valle, \& Hough, 1986). This questionnaire asks mothers to indicate how many days in the last week they have experienced symptoms using a 4-point Likert-type scale with scores ranging from 0 to 60 . A score of 16 or higher indicates the presence of significant levels of depressive symptoms (Vega et al.). Using this cut point, the CES-D, Spanish language version has shown acceptable sensitivity (.96) and specificity (.77) in identifying probable cases of Major Depressive Episode (MDE; Campo-Arias, Diaz-Martinez, Rueda-Jaimes, Cadena-Afanador, \& Hernandez, 2007; Cho et al., 1993). Both English and Spanish versions of the instrument 
have shown consistently adequate reliability among adult Mexican-Americans, Blacks and Anglos (Posner, Stewart, Marin, \& Perez-Stable, 2001; Roberts, Rhoades, \& Vernon, 1990; Roberts, Vernon, \& Rhoades, 1989) within Hispanic subgroups (Cho et al.) and concurrent and predictive validity (Cuijpers, Beekman, Smit, \& Deeg, 2006; Munet-Vilaro, Folkman, \& Gregorich, 1999; Roberts et al., 1989). In this study, Cronbach a for the CES-D ranged from .90 to .93 across the four time points. Mothers were paid $\$ 10$ for participating in the screening phase.

Mothers who scored $\geq 16$ on the CES-D were contacted by a bilingual research staff member and given a full explanation of the study's purpose, the random assignment process, and the data collection procedures. Interested mothers were screened for additional eligibility criteria and excluded if they were receiving psychotherapy or substance abuse counseling twice monthly or more often, or were taking a psychotropic medication. Mothers completed baseline data in their homes. Using a computer generated randomization list utilizing blocking and stratified by EHS site, participants were randomly assigned to either the intervention or a closely monitored, usual care condition. All mothers assigned to the usual care condition were offered the full intervention after their last data collection, or if they preferred, referral to services. The study was approved by the Institutional Review Board of the first author's university.

\section{Intervention}

Peplau (1952), in her interpersonal theory of nursing proposed that positive change for the client is the result of a skillfully constructed interpersonal process that is monitored and guided by the nurse. Peplau's theory provided the structure for the therapeutic relationship between nurses and mothers guiding delivery of the IPT-based protocol for the intervention (Weissman, 2007). Consistent with the original time-limited format of IPT, the protocol was limited to 16 contacts. The modifications made to the original IPT protocol for in-home delivery and the tailoring process for low-income English-speaking mothers and Latinas have been previously described (Beeber et al., 2004; Beeber, Perreira, \& Schwartz, 2008).

The intervention was hypothesized to help mothers modify the sources of their symptoms by linking their depressive symptoms to their origins in stressful interpersonal relationships, and to increase their self-efficacy by developing strategies to reduce symptoms, manage interpersonal stressors, improve social support, and provide responsive parenting. The mother's reports of aggression and negative social-emotional behaviors in the child were conceptualized as overcritical judgments that are part of the spectrum of depressive symptoms. As such, maternal reports of aggressive behaviors and concerns would be expected to decrease as symptoms abated. In addition, because the intervention emphasized parenting as one of the interpersonal problem areas, mothers would increase their responsiveness and developmental support through increased positive maternal interactions (Jones, Lamb-Parker, Schweder, \& Ripple, 2001; Love et al., 2005; Roggman, Newland, \& Cook, 2001).

The intervention content focused on four interpersonal problem areas: (a) reduction of depressive symptoms and their interpersonal sources, (b) choice and use of social support, (c) management of stressful life issues and interpersonal disputes, and (d) development of 
strategies to increase responsiveness to the child. Three strategy organizers or skill sheets were developed for each of the four interpersonal problem areas. Each skill sheet focused on a situation or interpersonal problem empirically derived from the narratives of symptomatic Latina mothers (Beeber et al., 2008). The mother chose one skill sheet that fit her circumstances from each of the four problem areas (four in total), and all mothers received a fifth skill sheet on strategies to combat depressive symptoms that recurred. To achieve a minimum dose, intervention nurses focused on each of the five skill sheets at least once during the intervention.

The intervention was delivered by teams of English-speaking master's-prepared psychiatric nurses and project-trained Spanish language interpreters (Beeber, Lewis, Cooper, Maxwell, \& Sandelowski, 2009). Two-thirds of the project-trained interpreters were bilingual EHS staff, who were already working with the mother. The nurse-interpreter team completed 11 in-home sessions interspersed with five short booster visits by the interpreter alone. After three consecutive visits by the nurse-interpreter team for assessment, formulation of the plan, and initiation of the intervention, the weekly team visit alternated with a 15-minute booster visit or telephone call the following week. The booster visits or calls were inserted between team visits to give mothers an opportunity to apply what they had learned during the team visits and allow EHS staff to complete their program-mandated work with mothers. The nurse worked closely with the interpreter to plan the booster visit, which focused on reviewing the skill sheets used in the previous team visit. After completing 16 contacts, the team concluded the intervention and helped the mother plan how to maintain her gains. These included arrangements for continuing services if needed. If the mother wished, her spouse/partner was invited to participate in the intervention visits; approximately $10 \%$ of spouse/partners met at least once with the nurse, interpreter, and the mother.

Prior to working with mothers, nurses completed 20-30 hours of classroom and supervised field training that included learning to work in the home setting, working with an interpreter, and sensitization to Latino cultural practices. The interpreters were formally trained by the bilingual project manager. A consecutive, conduit model of interpreting, in which the interpreter repeated what was said in the source language as exactly as possible in the target language, and relayed as closely as possible the emotional tone and emphasis of the target statements, was used to keep the nurse's therapeutic intention and adherence to the study protocol as accurate as possible (Baczynskyj, 2001; Bancroft, 2005). Most of the nurseinterpreter teams practiced together under the supervision of the PI and project manager prior to working with mothers. Nurses and interpreters were trained to de-brief together following each session, as many of the Latina interpreters had strong emotional responses to the sessions. Throughout the project, nurses discussed their work with each mother on a weekly telephone conference call with the PI and another team member, both of whom were experienced advanced practice psychiatric nurses. The nurses also completed logs that included objective data and a detailed narrative note of each visit.

All mothers in both groups completed questionnaires and were videotaped for 45-minutes interacting with their child in an unstructured manner, at baseline (T1), 14 weeks postbaseline or mid-intervention (T2), 22 weeks at termination of the intervention (T3), and 26 weeks or 1 month post intervention (T4). Prior randomized trials conducted with low- 
income mothers have had high attrition, which limited the analysis of intervention effects (Appleby et al., 1997; Spinelli \& Endicott, 2003). Therefore we designed a relatively short time frame (1 month) in which to measure post-intervention effects in order to retain as many mothers as possible in both the intervention and usual care conditions.

Most of the data collection was done in the mothers' homes. Responding to reports that instruments with ordinal response sets had not been answered accurately by Latinos (Flaskerud, 1988; McQuiston, Larson, Parrado, \& Flaskerud, 2002), we taught the mothers how to answer the questions in an ordinal response format at the first data collection visit. In addition, as the data collector read aloud each instrument and possible responses in Spanish, mothers held a small notebook containing the ordinal response set for that instrument to help them answer or point to the answer. These procedures allowed the inclusion of mothers who had low literacy in Spanish and English, and resulted in little missing data and very high internal consistency indices for the instruments. Mothers were paid an additional $\$ 10$ for each of the four data collection visits.

Throughout the study, all mothers received letters thanking them for participating and periodic cards and small gifts along with reminders of their next data collection time. At the second, third, and fourth data collection sessions, the mothers received framed photographs of themselves and their children taken during the previous visit. At the final session, all mothers were given a certificate of participation, and mothers in the usual care condition were offered the intervention.

Twenty-three of the mothers in the usual care group accepted the intervention after completing their last data collection, and the remaining mothers were given information about local mental health resources and assistance in making contact if they wished.

\section{Instruments}

Data were collected using interview questions and well-established questionnaires. Table 2 presents the descriptive statistics at each time point for the intervention and usual care mothers. All of the questionnaires were short, written in language that was not complex, and were published in a Spanish version that had shown acceptable reliability and validity with Spanish-speaking respondents. Prior to use, Spanish-speaking mothers from EHS who were similar to the target mothers evaluated the instruments for conceptual and linguistic clarity.

Maternal depressive symptom severity was measured at all four time points using the CESD, Spanish version, described earlier.

Maternal reports of aggression and concerns about behavior in the child were measured at Time 1 and Time 4 by the 19-item Child Behavior Checklist (CBCL), Spanish version, Child Aggression subscale (Achenbach, 1991) and the Ages and Stages QuestionnaireSocial-Emotional (ASQ-SE), Spanish version (Squires, Bricker, \& Twombly, 2002). Both instruments measure parental reports of behavioral problems; scores were used to represent overcritical judgments of the child's behaviors, a function of depressive symptoms. The 19item CBCL, which uses a 3-point Likert-type scale and yields scores ranging from 0 to 57 , is optimally used with children who are 18 months old or older and has stable psychometric 
properties. Test-retest reliability has been reported as $0.95-1.00$ with a Cronbach coefficient a of .78-.97 (Achenbach). In 2- 4-year-old African American, Latino, and White children $(N=682)$, the CBCL showed a high convergence with the Eyberg Child Behavior Inventory (Gross et al., 2007). Mothers with depressive symptoms have reported high levels of aggression on the CBCL without corroborating behaviors being observed in their children, suggesting that the parental CBCL score was elevated due to the symptomatology (WebsterStratton \& Hammond, 1988; Wolke \& Kuertjens, 1998). Cronbach a for the CBCL subscale in this study were .92 and .93 at $\mathrm{T} 1$ and $\mathrm{T} 4$, respectively.

The ASQ-SE is a field assessment instrument that uses maternal reports to assess social and emotional competence. The instrument is unique in having a standard scale for socialemotional functioning for children from 6 to 60 months. The mother is asked whether she has concerns about her child's emotional or social competence using a standard format that captures change over time. In a diverse population, the average coefficient a was .82 and test-retest reliability was .94; sensitivity was $78 \%$ and specificity was $94 \%$ (Squires et al., 2002). Mothers' concerns about the behaviors of the child were summed at each data point and used to represent maternal judgments of the child's behaviors.

Maternal responsiveness to the child was assessed with the Maternal-Child Observation (MCO) and the Home Observation for Measurement of the Environment (HOME) inventory. The MCO consists of maternal and child behaviors coded every 10 seconds by trained videocoders who were blinded to group membership. Further details on coding procedures are available (Holditch-Davis, Schwartz, Black, \& Scher, 2007). Five maternal behaviors that have been empirically observed to be altered in mothers with depressive symptoms were used for analysis: talking to the child, positive expressed affect, negative expressed affect, touching the child, and eye-to-eye contact. Spanish-speaking and nonSpanish-speaking coders were used. When non-Spanish speakers coded a tape, a native Latina bilingual coder reviewed the coding of all verbal behaviors to assure accurate interpretation. All coders achieved adequate inter-rater reliability (more than $85 \%$ exact agreement, and kappas exceeding .70) throughout the project. Cohen's $k s$ for this study ranged from .66 to .95 with a mean of .83 . The MCO has been used with 3-year-old prematurely born children, 6- and 18-month premature infants, medically fragile infants from birth to 1 year, and 12-24-month-old toddlers of HIV infected mothers (HolditchDavis, 2002; Holditch-Davis, Beeber, Mendoza, Schwartz, \& Perreira, 2007; Holditch-Davis et al., 2007).

The 0-3 version of the HOME Inventory (Caldwell \& Bradley, 1980) was designed to identify the degree of developmental stimulation present in the child's environment with higher scores representing a more affirming and stimulating environment. The 45-item HOME is scored dichotomously (present-absent) for a range of scores from 0 to 45 . The HOME was scored by the data collector using observational data and a non-directive interview of the mother. The HOME has been correlated with the developmentally supportive behaviors of mothers (Holditch-Davis, Tesh, Goldman, Miles, \& D'Auria, 2000; Tesh \& Holditch-Davis, 1997) and with later school performance (Bradley, 1987; Bradley et al., 1994; Caldwell \& Bradley) in ethnically and socioeconomically diverse samples (Bradley et al.; Tesh \& Holditch-Davis). Test-retest reliabilities for the total HOME scale, 
when scored at 6 and 12-month intervals, have been .76-.77 (Caldwell \& Bradley; HolditchDavis et al.; Tesh \& Holditch-Davis). Convergent validity has been shown with the Home Screening questionnaire (Frankenburg \& Coons, 1986). In this study, Cronbach as for the total HOME scale were $.86, .78, .81$, and .86 for T1-T4 respectively.

One mediator, maternal self-efficacy, was measured at all four time points using the 20-item General Self-Efficacy Scale (GSE), Spanish version (Luszczynska, Scholz, \& Schwarzer, 2005; Schwarzer \& Born, 1997). The GSE has 20 items that use a 4-point Likert-type scale. Items are summed to yield a final composite score with a range from 20 to 80 . Criterion validity has been demonstrated through positive correlations with optimism and work satisfaction and negative correlations with depression, anxiety, stress, and burnout (Luszczynska et al.). Cronbach as for the GSE ranged from .90 to .93 across the four time points.

Demographic moderators (age, education, partnered status, number of children under the age of 5 years) were measured at baseline using some items from the EHS national evaluation study (Beeber et al., 2007). Other moderators included acculturation, health status, and maternal burden factors. Acculturation was measured by the 4-item version of the Short Acculturation Scale for Hispanics (SASH) and the 10-item Psychological Acculturation Scale (PAS). The SASH scale yields scores between 5 and 20 and has shown high reliability and validity (Marin, Sabogal, Marin, Otero-Sabogal, \& Perez-Stable, 1987). The SASH was also used to determine that mothers had limited English language proficiency, as indicated by their low score on the four language items from the SASH (on average, $1.2[S D=0.5]$ ). A score of less than 2.9 indicates low language acculturation (Y. Jackson, 2006). Cron-bach's as in this study for the SASH ranged from .80 to .92 across the four time points. The PAS is scored 1-3 on an ordinal scale, with higher scores indicating greater acculturation. The PAS assesses the individual's sense of psychological attachment to Anglo-American and Latino/Hispanic cultures; respondents whose mean score is between 1.5 and 2.5 are considered bicultural, with individuals below and above that range considered less or more fully acculturated. The PAS has shown excellent internal consistency (Cronbach's $\alpha=.91$ ) in previous studies (Tropp, Coll, Alarcon, Vazquez-Garcia, 1999). Cronbach's as for the PAS ranged from .83 to .88 across the four time points.

Maternal health was measured using the Medical Outcomes Study Short Form Health Survey (SF-12), Spanish version (Gandek et al., 1998), a 12-item scale with subscales representing physical and emotional health and role functioning, pain, vitality, and social functioning. Scores range from 12 to 56. Cronbach's as have been reported from .78 (pain and general health) to .93 (physical functioning). In this sample, Cronbach's as for the SF-12 ranged from .80 to .88 across the four time points. Maternal burden factors, including dependence on others for transportation, child health problems, child use of disability services, legal problems, and level of family conflict, were obtained by interview questions and scored as present or absent.

\section{Statistical Analysis}

Data were entered into SAS databases and verified via double entry prior to analysis. The primary analysis to determine efficacy of the intervention was a repeated measures, general 
linear mixed-effects model for the CES-D scores. The effect of the intervention versus usual care groups at each follow-up time point relative to baseline was estimated, and multiple comparisons across time points were controlled through the use of the Hochberg method (Hochberg, 1988), a less conservative counterpart to the Bonferroni correction. A supportive analysis was conducted across the three follow-up timepoints in which the baseline (T1)

CES-D score was used as a covariate, in order to control for possible random imbalances in CES-D between the groups at baseline.

Similar analytic strategies were implemented for the coded videotaped maternal interaction behaviors (with additional covariate adjustment for the child's age), as well as the HOME subscale and total scores. Other analyses on change scores between one follow-up time and at baseline utilized two-group, independent samples $t$-tests to evaluate differences between the intervention and usual care group means. Pearson correlation coefficients (ordinary and partial) between change variables for CES-D and GSE between T3 and T1 were computed and tested to assess mediation. Moderators were assessed separately through the addition of an interaction term involving each moderator and intervention group in linear regression models for the change in CES-D from T1 to T3.

\section{RESULTS}

\section{Impact of the Intervention on Maternal Depressive Symptom Severity}

The repeated measures modeling analysis showed a significantly greater decrease in mean depressive symptom severity scores (CES-D) in the intervention group than the usual care group at each time point. The mean differences (with standard errors) were: Time 2 ( -6.8 [2.9] points, $p=.02$ ), Time 3 ( -10.6 [3.3] points, $p<.01$ ), and Time 4 [-8.4 (3.6) points, $p$ $=.02]$; see Fig. 2) The intervention group significantly improved by Time $2(p<.001)$ with the mean symptom level below the critical cut off of 16 on the CES-D with further decreases at each subsequent time point.

To ascertain whether the higher baseline scores on the CES-D in the intervention group inflated the change scores, the supportive repeated measures analysis conducted across the follow-up period and using baseline (T1) value as a covariate also showed improvement in CES-D over the control group at each of the follow-up times. These mean differences (with standard errors) were $-4.1(2.3)$ points $[p=.08]$ at $\mathrm{T} 2,-8.3(2.3)$ points $[p<.01]$ at $\mathrm{T} 3$, and $-6.1(2.5)$ points $[p=.04]$ at $\mathrm{T} 4$.

Another way of portraying these changes in the intervention and usual care groups was to compare the number of mothers scoring at or above the CES-D cut point (16) at each time point. At T1,74\% of mothers in the intervention group were at or above 16 compared to $71 \%$ in the usual care group; at T2, 45\% (intervention) compared to 56\% (usual care); at T3, $30 \%$ (intervention) compared to $69 \%$ (usual care); and at T4, $22 \%$ of intervention mothers were at or above the cut point compared to $47 \%$ of the usual care mothers. 


\section{Impact of the Intervention on Maternal Reports of Aggression in the Child and Concerns about Social-Emotional Competence}

Eighteen mothers had children old enough to complete the aggression subscale of the CBCL at the first and last data collections (10 intervention/8 usual care). Mothers in the intervention group significantly reduced their mean scores on reports of aggression between Time 1 and Time 4 ( -5.5 points) compared to an increase (4.3 points) in the usual care group $(t=2.43, d f 13.9, p=.03)$. Mothers' concerns about their children's social and emotional competence (ASQ-SE) did not differ significantly between the two groups. However, the direction of maternal concerns differed. The intervention group mean dropped (-.40, $S D=$ 1.8; indicating fewer concerns about the child's social-emotional behaviors); the usual care group increased $(.48, S D=1.0)$ between $\mathrm{T} 1$ and $\mathrm{T} 4$, indicating concerns about their children's problematic social-emotional behaviors increased in the usual care condition. Despite the small sample (3 intervention boys vs. 6 usual care boys), when we analyzed the scores by gender, we found nearly two units of change in mothers' reports of the behaviors of the boys between T1 and T4 $(-1.4[S D=2.8]$ intervention boys vs. $0.6[S D=1.2]$ usual care boys). Analyses of the coded videotaped maternal interaction behaviors showed no significant differences in the interaction behaviors of intervention and usual care mothers or their children. Comparison of the HOME subscale scores and total HOME scores showed no significant differences between intervention and usual care groups.

\section{Increased Self-efficacy as Mediator of the Intervention Effect}

To heuristically determine the extent of mediation, we plotted the CES-D (depression severity) and GSE (general self-efficacy) scores across time for the intervention mothers; scores followed the hypothesized pattern of increased GSE paralleling decreased CES-D scores (see Fig. 3). Partial correlations were used to examine whether self-efficacy exerted a mediating effect on CES-D at T3, the end of the intervention, and before the postintervention period, when the largest observed effect was expected to occur (Baron \& Kenny, 1986). In a fully mediated model, we would expect the relationship between the intervention group and change in CES-D (Baron \& Kenny, 1986) from enrollment $(r=-.40$, $p<.01)$ to drop to near-zero when the effect of change in self-efficacy from enrollment was removed from both intervention group and change in CES-D. Instead, when the GSE effect was removed, the correlation between the intervention group and change in CES-D diminished slightly $(r=-.35)$ but remained statistically significant $(p<.01)$, indicating that increasing maternal self-efficacy only partially mediated the effect of the intervention on lowering CES-D scores (see Fig. 4).

\section{Moderators of the Intervention Effect}

The sample size limited the statistical power of the analyses of moderators; however, physical health and function (SF-12, Spanish version) in the mothers showed a significant interaction with group membership and depression $(p=.01)$. In order to remove potential confounding with the CES-D, the one depression item was deleted from the SF-12 and the results were similar $(p<.01)$. These results indicated that mothers with lower SF-12 scores (poorer physical health) benefited more from the intervention (greater decrease in CES-D scores from T1 to T3) than mothers with higher SF-12 scores $(t=2.6, \mathrm{df}=1, p=.01)$ 
Intervention mothers $1 S D$ below the mean on physical health would be expected to experience a 25 point drop $(\mathrm{SE}=3.1, p<.001)$ in the CES-D at $\mathrm{T} 3$, while intervention mothers $1 S D$ above the mean, as well as control mothers $1 S D$ above or below the mean experienced would be expected to have drops of 5.5, 3.1, and 4.3 points in CES-D, respectively (all $p>.10$ ). Using the unstandardized regression estimate, for every unit of change in reported health and function, the difference in CES-D between the intervention and usual care groups increased by 1.5 points. Thus, mothers with lower SF-12 scores (poorer physical health) benefited more from the intervention (greater decrease in CES-D scores from T1 to T3) than mothers with higher SF-12 scores. No other moderators showed significant interactions with the group membership of the mothers.

\section{DISCUSSION}

The intervention showed efficacy in producing short-term reduction of depressive symptoms with a mean CES-D score for the intervention group well below the critical cut score of 16 at the T2 measurement point. The intervention group mean continued to decrease at each subsequent time point even after completing the intervention and stopping contact with the nurse for 1 month. The greater decrease in mean CES-D scores in the intervention group compared to the usual care group was consistent with the pattern we expected to achieve through intensive intervention, and suggests that the intervention was successful in shortening the length of time these infants and toddlers were exposed to maternal depressive symptoms. However, the usual care group's mean CES-D also decreased. This decline may have been a result of close monitoring by the research team, and the support that all mothers received through EHS. It is important to note that this decline resulted in a usual care group mean only slightly below the critical cut point of 16 , indicating that these mothers averaged significant levels of symptoms at three of the four observation points. As noted before, the length of time the child is exposed to symptoms is critical during this era of rapid development.

Despite the small number of mothers with children old enough to complete the CBCL at both time points, the significant decrease in the intervention group mothers' reports of aggression in the child that accompanied the reduction in depressive symptoms may have important implications as well. Some of the parenting components of the intervention were directed at helping mothers change their highly critical perceptions of themselves and, in turn, their negative perceptions of their children. As Forman et al. (2007) observed, improvement in the mother's perceptions of the child could potentially improve the child's view of him/herself, which subsequently would affect learning, social behavior, and emotional regulation.

The effect of the intervention on depressive symptoms appeared to be only partially mediated by self-efficacy, which may indicate that other factors such as social support, instrumental resources, and other benefits of EHS programming also contributed. An encouraging finding was that the intervention was particularly effective for mothers with compromised physical health, who were the least mobile and most highly vulnerable participants. 
The lack of statistically significant differences between the intervention and usual care mothers in maternal interaction and responsiveness on the MCO and HOME was unexpected. In a related comparison of mothers in this study with asymptomatic Latina mothers with limited English proficiency recruited from the same three EHS programs at the same time, significant differences were observed on the MCO at baseline (Beeber et al., 2008; Perreira et al., 2009) Asymptomatic mothers looked at their infants or toddlers and had eye-to-eye contact more often, talked more frequently, and were more elaborative in their play than symptomatic mothers. When compared on the HOME, symptomatic mothers provided fewer stimulating activities and were less involved with their infants and toddlers than asymptomatic mothers. Two reasons that there were no effects of the intervention for the symptomatic mothers in the current study may have been that the time period (1 month) between the end of the intervention and the final data collection was too short for the intervention mothers to make substantial change in their interaction patterns, or that the parenting guidance provided by EHS to both groups was sufficiently strong to remove the group differences over the 6-month time frame. We will explore these findings with subsequent studies.

The study confirmed that in this sample of EHS Latina mothers depressive symptoms, as assessed by the CES-D, were prevalent and severe. Although we cannot generalize from this convenience sample, the percentage of mothers with significant symptom severity (57\%) was higher than other studies (Alderete et al., 2000; Riolo et al., 2005) and replicated the high prevalence reported in the EHS evaluation study (48\%) that used a large national sample (Administration for Children and Families Office of Planning Research \& Evaluation - 2006). A strength of this study was the high enrollment rate (87\% of eligible mothers), which we attributed to our partnership with EHS and the high trust that mothers had in the bilingual staff and home visitors. The retention rate in the intervention (87\%) was also impressive in comparison to prior studies. We attributed this to the in-home model of delivery, the careful tailoring of the intervention, and to the skill and persistence of the PMH nurses and their interpreter teammates. In the course of the study, our EHS staff partners described the difficulties they encountered in providing the EHS-mandated parenting guidance to symptomatic mothers. In the national evaluation of EHS, mothers with depressive symptoms and high stressors had difficulty meeting attendance requirements and withdrew from EHS prematurely (Administration for Children and Families Office of Planning Research and Evaluation 2002). We can surmise that reduction of depressive symptoms through an intervention such as that used in the current study may have an added benefit of helping symptomatic mothers remain in EHS and use the enrichment resources, which could in turn benefit their vulnerable infants and toddlers.

The findings for the efficacy of the intervention are limited by the significant results occurring only in the self-report measures. In addition, we did not gather DSM-IV diagnostic information on how many of these Latina mothers met criteria for Major Depressive Episode, which would have helped us identify more precisely whether the intervention was more or less effective for mothers with more severe symptoms. Our analysis of mediators and moderators was limited by the sample size, and thus the mediating role of self-efficacy should be explored further in a larger sample. Finally, we do not know whether the mothers' gains held past the 1-month post-intervention period. 
This study of a group isolated by language differences with highly vulnerable children used a pool of existing mental health professionals (psychiatric mental health advanced practice nurses) and available community providers (EHS staff) in a new destination area where resources are scarce for mothers with limited English proficiency (Alpert, 2005). The advanced practice psychiatric nurses were able to engage these symptomatic mothers and carry out the intervention with minimal additional training, despite reliance on an interpreter and differences in cultural and socioeconomic backgrounds. These findings indicate that a pressing need for mental health intervention can be addressed by reconfiguring existing resources, an advantage when resources are scarce.

Our efforts to involve low-literacy mothers in the intervention and data collection processes were also successful. In exit interviews, mothers said that the intervention fit their needs well. Although pairing the nurse with an interpreter was a model developed to meet immediate health disparities for mothers in a new destination area, the resulting system offers promise as a model that can be adapted to other areas with shortages of multilingual providers. The success of the intervention in reducing depressive symptoms represents a promising approach toward reducing this serious threat to infant-toddler mental health. By imbedding the intervention in an existing community program (EHS), the model may serve as an example for designing mental health interventions with other high risk populations for whom traditional mental health services are not available or acceptable.

\section{Acknowledgments}

This study was supported by funding from DHHS/Administration for Children and Families/Early Head Start University Partnership grant 90YF0042 (Beeber, PI) and Young Scholars Program of the Foundation for Child Development (Perreira, PI). We are grateful for the help of the participating mothers and their families, our Early Head Start program partners, our research staff, and the intervention nurses- especially Sara L. Emory, RN, MSN, PMHCNS-BC and Carolyn Cooper, PHD, RN. We are indebted to Rachel Chazan Cohen, PhD and the E-PIRC Consortium members (Beeber et al., 2007), Sandy Funk, PhD, and Kathryn E. Barnard, PhD, RN.

\section{REFERENCES}

Achenbach, TM. Manual for the Child Behavior Checklist/4-18 and 1991 Profile. University of Vermont, Department of. Psychiatry; Burlington, VT: 1991.

Administration for Children and Families Office of Planning Research and Evaluation. Pathways to quality and full implementation in Early Head Start programs. Author; Washington, DC: 2002.

Administration for Children and Families Office of Planning Research \& Evaluation. Research to practice: Depression in the lives of Early Head Start families. Author; Washington, DC: 2006.

Alderete E, Vega WA, Kolody B, Aguilar-Gaxiola S. Effects of time in the United States and Indian ethnicity on DSM-III-R psychiatric disorders among Mexican Americans in California. The Journal of Nervous and Mental Disease. 2000; 188:90-100. [PubMed: 10695837]

Alpert, RT. [September 10, 2007] The New Latino South. 2005. from http://diversity.gsfc.nasa.gov/ dcprivate/diversityresources/new_latino_south.htm

American Psychiatric Association. Diagnostic and statistical manual of mental disorders. 4th ed., text revision ed.. Author; Washington, DC: 2000.

Appleby L, Warner R, Whitton A, Faragher B. A controlled study of fluoxetine and cognitivebehavioural counselling in the treatment of postnatal depression. BMJ. 1997; 314:932-936. [PubMed: 9099116]

Baczynskyj W. Language, culture, memory intertwined. Connections. 2001; 2:10-12.

Bancroft, M. The interpreter's world tour: An environmental scan of standards of practice for interpreters. The California Endowment; Woodland Hills, CA: 2005. 
Baron RM, Kenny DA. The moderator-mediator variable distinction in social psychological research: Conceptual, strategic, and statistical considerations. Journal of Personality and Social Psychology. 1986; 51:1173-1182. [PubMed: 3806354]

Beardslee WR, Gladstone TR. Prevention of childhood depression: Recent findings and future prospects. Biological Psychiatry. 2001; 49:1101-1110. [PubMed: 11430852]

Beeber LS, Chazen-Cohen R, Squires J, Harden BJ, Boris N, Scott-Heller S, et al. The Early Promotion and Intervention Research Consortium (E-PIRC): Five approaches to improving infant/ toddler mental health in Early Head Start. Infant Mental Health Journal. 2007; 28:130-150.

Beeber L, Holditch-Davis D, Belyea M, Funk SG, Canuso R. In-home intervention for depressive symptoms with low-income mothers of infants and toddlers in the United States. Health Care for Women International. 2004; 25:561-580. [PubMed: 15354622]

Beeber L, Lewis V, Cooper C, Maxwell L, Sandelowski M. Meeting the "now” need: PMH-APRNinterpreter teams provide in-home mental health intervention for depressed Latina mothers with limited English proficiency (LEP). Journal of the American Psychiatric Nurses Association. 2009; 15:249-259. [PubMed: 21665811]

Beeber LS, Perreira KM, Schwartz T. Supporting the mental health of mothers raising children in poverty: How do we target them for intervention studies? Annals of the New York Academy of Sciences. 2008; 1136:86-100. [PubMed: 17954677]

Black MM, Papas MA, Hussey JM, Dubowitz H, Kotch JB, Starr RH Jr. Behavior problems among preschool children born to adolescent mothers: Effects of maternal depression and perceptions of partner relationships. Journal of Clinical Child and Adolescent Psychology. 2002; 31:16-26. [PubMed: 11845646]

Black MM, Papas MA, Hussey JM, Hunter W, Dubowitz H, Kotch JB, et al. Behavior and development of preschool children born to adolescent mothers: Risk and 3-generation households. Pediatrics. 2002; 109:573-580. [PubMed: 11927698]

Boyd RC, Zayas LH, McKee MD. Mother-infant interaction, life events and prenatal and postpartum depressive symptoms among urban minority women in primary care. Maternal and Child Health Journal. 2006; 10:139-148. [PubMed: 16397831]

Bradley RH. The early development of low birth weight infants: Relationship to health, family status, family contest, family processes, and parenting. International Journal of Behavioral Development. 1987; 10:301-318.

Bradley RH, Mundfrom DJ, Whiteside L, Caldwell BM, Casey PH, Kirby RS, et al. A reexamination of the association between HOME scores and income. Nursing Research. 1994; 43:260-266. [PubMed: 7524040]

Brennan PA, Hammen C, Katz AR, Le Brocque RM. Maternal depression, paternal psychopathology, and adolescent diagnostic outcomes. Journal of Consulting and Clinical Psychology. 2002; 70:1075-1085. [PubMed: 12362958]

Breznitz Z, Sherman T. Speech patterning of natural discourse of well and depressed mothers and their young children. Child Development. 1987; 58:395-400. [PubMed: 3829785]

Caldwell, B.; Bradley, R. Home Observation for Measurement of the Environment. University of Arkansas at Little Rock; Little Rock, AR: 1980.

Campo-Arias A, Diaz-Martinez L, Rueda-Jaimes G, Cadena-Afanador L, Hernandez N. Psychometric properties of the CES-D Scale among Colombian adults from the general population. Revista Colombiana de Psiquiatra. 2007; 36:664-674.

Chabrol H, Teissedre F, Saint-Jean M, Teisseyre N, Roge B, Mullet E. Prevention and treatment of post-partum depression: A controlled randomized study on women at risk. Psychological Medicine. 2002; 32:1039-1047. [PubMed: 12214785]

Chaudron LH, Kitzman HJ, Peiper KL, Morrow S, Perez LM, Newman MC. Self-recognition of and provider response to maternal depressive symptoms in low-income Hispanic women. Journal of Women's Health. 2005; 14:331-338.

Cho MJ, Moscicki EK, Narrow WE, Rae DS, Locke BZ, Regier DA. Concordance between two measures of depression in the Hispanic Health and Nutrition Examination Survey. Social Psychiatry and Psychiatric Epidemiology. 1993; 28:156-163. [PubMed: 8235801]

Cohen, J. Statistical power analysis for the behavioral sciences. Academic Press; New York: 1977. 
Cohn JF, Matias R, Tronick EZ, Connell D, Lyons-Ruth K. Face-to-face interactions of depressed mothers and their infants. New Directions in Child Development. 1986; 34:31-45.

Cohn JF, Tronick E. Specificity of infants' response to mothers' affective behavior. Journal of the American Academy of Child and Adolescent Psychiatry. 1989; 28:242-248. [PubMed: 2925579]

Coyl DD, Roggman LA, Newland LA. Stress, maternal depression, and negative mother-infant interactions in relation to infant attachment. Infant Mental Health Journal. 2002; 23:143-163.

Cuijpers P, Beekman A, Smit F, Deeg D. Predicting the onset of major depressive disorder and dysthymia in older adults with subthreshold depression: A community based study. International Journal of Geriatric Psychiatry. 2006; 21:811-818. [PubMed: 16955441]

Field T. Maternal depression effects on infants and early interventions. Preventive Medicine. 1998; 27:200-203. [PubMed: 9578995]

Field T, Healy B, Goldstein S, Gutherz M. Behavior-state matching and synchrony in mother-infant interactions of nondepressed versus depressed dyads. Developmental Psychology. 1990; 26:7-14.

Finch BK, Kolody B, Vega WA. Perceived discrimination and depression among Mexican-origin adults in California. Journal of Health and Social Behavior. 2000; 41:295-313. [PubMed: 11011506]

Flaskerud JH. Is the Likert scale format culturally biased? Nursing Research. 1988; 37:185-186. [PubMed: 3368361]

Flores G, Abreu M, Tomany-Korman SC. Limited English proficiency, primary language at home, and disparities in children's health care: How language barriers are measured matters. Public Health Reports. 2005; 120:418-430. [PubMed: 16025722]

Flores G, Abreu M, Tomany-Korman SC. Why are Latinos the most uninsured racial/ethnic group of US children? A community-based study of risk factors for and consequences of being an uninsured Latino child. Pediatrics. 2006; 118:e730-e740. [PubMed: 16950964]

Flores G, Fuentes-Afflick E, Barbot O, Carter-Pokras O, Claudio L, Lara M, et al. The health of Latino children: Urgent priorities, unanswered questions, and a research agenda. JAMA: The Journal of the American Medical Association. 2002; 288:82-90. [PubMed: 12090866]

Flores G, Vega LR. Barriers to health care access for Latino children: A review. Family Medicine. 1998; 30:196-205. [PubMed: 9532442]

Forman DR, O'Hara MW, Stuart S, Gorman LL, Larsen KE, Coy KC. Effective treatment for postpartum depression is not sufficient to improve the developing mother-child relationship. Developmental Psychopathology. 2007; 19:585-602.

Frankenburg WK, Coons CE. Home Screening Questionnaire: Its validity in assessing home environment. The Journal of Pediatrics. 1986; 108:624-626. [PubMed: 3958839]

Gandek B, Ware JE, Aaronson NK, Apolone G, Bjorner JB, Brazier JE, et al. Cross-validation of item selection and scoring for the SF-12 Health Survey in nine countries: Results from the IQOLA Project. International Quality of Life Assessment. Journal of Clinical Epidemiology. 1998; 51:1171-1178. [PubMed: 9817135]

Goodman SH, Brogan D, Lynch ME, Fielding B. Social and emotional competence in children of depressed mothers. Child Development. 1993; 64:516-531. [PubMed: 8477632]

Gotlib IH, Lewinsohn PM, Seeley JR. Symptoms versus a diagnosis of depression: Differences in psychosocial functioning. Journal of Consulting and Clinical Psychology. 1995; 63:90-100. [PubMed: 7896995]

Gross D. Implications of maternal depression for the development of young children. Image: The Journal of Nursing Scholarship. 1989; 21:103-107. [PubMed: 2471684]

Gross D, Fogg L, Young M, Ridge A, Cowell J, Sivan A, et al. Reliability and validity of the Eyberg Child Behavior Inventory with African-American and Latino parents of young children. Research in Nursing \& Health. 2007; 30:213-223. [PubMed: 17380522]

Hammen, C. Depression runs in families. Springer-Verlag; New York: 1991.

Hochberg Y. A sharper Bonferroni procedure for multiple tests of significance. Biometrika. 1988; 75:800-802.

Holditch-Davis, D. Methodological issues in systematic observation.. Presented at the 16th annual meeting of the Southern Nursing Research Society; San Antonio, TX. Feb. 2002 
Holditch-Davis, D.; Beeber, L.; Mendoza, Z.; Schwartz, T.; Perreira, K. Coding techniques for observations of interactions of Spanish-speaking mothers with their young children.. Presented at the biennial meeting of the Society for Research on Child Development; Boston. Mar. 2007

Holditch-Davis D, Schwartz T, Black B, Scher M. Correlates of mother-premature infant interactions. Research in Nursing \& Health. 2007; 30:333-346. [PubMed: 17514707]

Holditch-Davis D, Tesh EM, Goldman BD, Miles MS, D'Auria J. Use of the HOME Inventory with medically fragile infants. Children's Health Care. 2000; 29:257-278.

Jackson A, Brooks-Gunn J, Huang C, Glassman M. Single mothers in low-wage jobs: Financial strain, parenting, and preschoolers' outcomes. Child Development. 2000; 71:1409-1423. [PubMed: 11108104]

Jackson, Y. Encyclopedia of multi-cultural psychology. Sage; Thousand Oaks, CA: 2006. Acculturation scales: Short Acculturation Scale for Hispanics.; p. 16-17.

Jones, SM.; Lamb-Parker, F.; Schweder, A.; Ripple, C. Parent involvement in HeadStart: Context and consequences. Columbia University; New York: 2001.

Kessler RC, Merikangas KR, Berglund P, Eaton WW, Koretz DS, Walters EE. Mild disorders should not be eliminated from the DSM-IV. Archives of General Psychiatry. 2003; 60:1117-1122. [PubMed: 14609887]

Keyes L, Muller K. IML Power Program Software developed under NICHD Mental Retardation Research Grant P30-HDO-3110-22. 1991

Kisker, E.; Love, J.; Paulsell, D.; Raikes, H. Leading the way: Characteristics and early experiences of selected Early Head Start Programs. US Department of Health and Human Services; Washington, D.C.: 2000 .

Kochanska G, Radke-Yarrow M, Kuczynski L, Friedman SL. Normal and affectively ill mothers' beliefs about their children. American Journal of Orthopsychiatry. 1987; 57:345-350. [PubMed: 3618732]

Love JM, Kisker EE, Ross C, Raikes H, Boller K, et al. The effectiveness of early head start for 3year-old children and their parents: Lessons for policy and programs. Developmental Psychology. 2005; 41:885-901. [PubMed: 16351335]

Lovejoy MC, Graczyk PA, O'Hare E, Neuman G. Maternal depression and parenting behavior: A meta-analytic review. Clinical Psychology Review. 2000; 20:561-592. [PubMed: 10860167]

Luszczynska A, Scholz U, Schwarzer R. The general self-efficacy scale: multicultural validation studies. Journal of Psychology. 2005; 139:439-457. [PubMed: 16285214]

Marin G, Sabogal F, Marin BV, Otero-Sabogal R, Perez-Stable EJ. Development of a Short Acculturation Scale for Hispanics. Hispanic Journal of Behavioral Sciences. 1987; 9:183-205.

Marrow HB. New destinations and immigrant incorporation. Perspectives on Politics. 2005; 3:781799.

McQuiston C, Larson K, Parrado EA, Flaskerud JH. AIDS knowledge and measurement considerations with unacculturated Latinos. Western Journal of Nursing Research. 2002; 24:354372. [PubMed: 12035910]

Miller, S. [November 16, 2007] Hispanics replace African Americans as largest U.S. minority group. 2003. from http://www.america.gov/st/washfile-english/2003/January/ 20030123174627smiller@pd.state.gov0.1734735.html

Miranda J, Bernal G, Lau A, Kohn L, Hwang W-C, LaFromboise T. State of the science on psychosocial interventions for ethnic minorities. Annual Review of Clinical Psychology. 2005; 1:113-142.

Moher D, Schulz KF, Altman D. The CONSORT Statement: Revised recommendations for improving the quality of reports of parallel-group randomized trials 2001. Explore (NY). 2005; 1:40-45. [PubMed: 16791967]

Munet-Vilaro F, Folkman S, Gregorich S. Depressive symptomatology in three Latino groups. Western Journal of Nursing Research. 1999; 21:209-224. [PubMed: 11512178]

Murray L, Fiori-Cowley A, Hooper R, Cooper P. The impact of postnatal depression and associated adversity on early mother-infant interactions and later infant outcome. Child Development. 1996; 67:2512-2526. [PubMed: 9022253] 
Nadeem E, Lange JM, Edge D, Fongwa M, Belin T, Miranda J. Does stigma keep poor young immigrant and US-born black and Latina women from seeking mental health care? Psychiatric Services. 2007; 58:1547-1554. [PubMed: 18048555]

Needlman R, Stevenson J, Zuckerman B. Psychosocial correlates of severe temper tantrums. Journal of Behavioral and Developmental Pediatrics. 1991; 12:77-83.

Peplau, H. Interpersonal relations in nursing. Springer; New York: 1952.

Perreira, K.; Beeber, LS.; Schwartz, T.; Holditch-Davis, D.; Ornelas, I.; Maxwell, L. Preparing the way: Early Head Start and the socio-emotional health of infants and toddlers in Latino immigrant families.. In: Grigorenko, E.; Takanishi, R., editors. Immigration, diversity, and education. Routledge/Taylor and Francis; New York: 2009. p. 67-94.

Posner SF, Stewart AL, Marin G, Perez-Stable EJ. Factor variability of the Center for Epidemiological Studies Depression Scale (CES-D) among urban Latinos. Ethnicity and Health. 2001; 6:137-144. [PubMed: 11488294]

Radke-Yarrow, M.; Belmont, B.; Nottelmann, E.; Bottomly, L. Young children's selfconceptions: Origins in the natural discourse of depressed and normal mothers and their children. The self in transition.. In: Cicchetti, D.; Beeghly, M., editors. The self in transition: Infancy to childhood. University of Chicago; Chicago: 1990. p. 345-361.

Radke-Yarrow M, Nottelmann E, Belmont B, Welsh JD. Affective interactions of depressed and nondepressed mothers and their children. Journal of Abnormal Child Psychology. 1993; 21:683695. [PubMed: 8126320]

Radloff LS. The CES-D Scale: A self-report depression scale for research in the general population. Applied Psychological Measurement. 1977; 1:285-401.

Riolo SA, Nguyen TA, Greden JF, King CA. Prevalence of depression by race/ethnicity: Findings from the National Health and Nutrition Examination Survey III. American Journal of Public Health. 2005; 95:998-1000. [PubMed: 15914823]

Roberts RE, Rhoades HM, Vernon SW. Using the CES-D scale to screen for depression and anxiety: Effects of language and ethnic status. Psychiatry Research. 1990; 31:69-83. [PubMed: 2315423]

Roberts RE, Vernon SW, Rhoades HM. Effects of language and ethnic status on reliability and validity of the Center for Epidemiologic Studies-Depression Scale with psychiatric patients. The Journal of Nervous and Mental Disease. 1989; 177:581-592. [PubMed: 2677237]

Roggman, L.; Newland, L.; Cook, G. Home visits and parents' mental health: Developmental and contextual transitions of children and families. Columbia; New York: 2001.

Rosenblum O, Mazet P, Bénony H. Mother and infant affective involvement states and maternal depression. Infant Mental Health Journal. 1997; 18:350-363.

Ruttenberg W, Finello K, Cordeiro AK. Interactions between depressed and nondepressed Latina mothers and their premature infants. Infant Mental Health Journal. 1997; 18:364-377.

Schwarzer R, Born A. Optimistic self-beliefs: Assessment of general perceived self efficacy in thirteen cultures. World Psychology. 1997; 3:177-190.

Sherbourne CD, Wells KB, Hays RD, Rogers W, Burnam MA, Judd LL. Subthreshold depression and depressive disorder: Clinical characteristics of general medical and mental health specialty outpatients. American Journal of Psychiatry. 1994; 151:1777-1784. [PubMed: 7977885]

Spinelli MG, Endicott J. Controlled clinical trial of interpersonal psychotherapy versus parenting education program for depressed pregnant women. American Journal of Psychiatry. 2003; 160:555-562. [PubMed: 12611838]

Squires, J.; Bricker, D.; Twombly, L. Ages and Stages Questionnaires: Social-Emotional (ASQ-SE). Brookes; Baltimore: 2002.

Stuart S, O'Hara MW. Treatment of postpartum depression with interpersonal psychotherapy. Archives of General Psychiatry. 1995; 52:75-76. [PubMed: 7811164]

Stuart S, O'Hara MW, Gorman LL. The prevention and psychotherapeutic treatment of post-partum depression. Archives of Women's Mental Health. 2003; 6:S57-S69.

Tesh EM, Holditch-Davis D. HOME inventory and NCATS: Relation to mother and child behaviors during naturalistic observations. Home Observation for Measurement of the Environment. Research in Nursing \& Health. 1997; 20:295-307. [PubMed: 9256876] 
Tropp LR, Coll CG, Alarcon O, Vazquez-Garcia HA. Psychological acculturation: Development of a new measure for Puerto Ricans on the U.S. mainland. Educational and Psychological Measurement. 1999; 59:351-367. [PubMed: 21415932]

Vega WA, Kolody B, Aguilar-Gaxiola S, Catalano R. Gaps in service utilization by Mexican Americans with mental health problems. American Journal of Psychiatry. 1999; 156:928-934. [PubMed: 10360134]

Vega WA, Kolody B, Valle R, Hough R. Depressive symptoms and their correlates among immigrant Mexican women in the United States. Social Science \& Medicine. 1986; 22:645-652. [PubMed: 3715504]

Webster-Stratton C, Hammond M. Maternal depression and its relationship to life stress, perceptions of child behavior problems, parenting behaviors, and child conduct problems. Journal of Abnormal Child Psychology. 1988; 16:299-315. [PubMed: 3403812]

Weissman MM. Recent non-medication trials of interpersonal psychotherapy for depression. International Journal of Neuropsychopharmacology. 2007; 10:117-122. [PubMed: 16787556]

Weissman, MM.; Markowitz, JC.; Klerman, GL. Comprehensive guide to interpersonal psychotherapy. Basic; New York: 2000.

Wickberg B, Hwang CP. Counseling of postnatal depression: A controlled study on a population based Swedish sample. Journal of Affective Disorders. 1996; 39:209-216. [PubMed: 8856425]

Wolke D, Kuertjens S. Maternal depression and cognitive and behavioral development over the first 9 years of life. Infant Behavior \& Development. 1998; 21:763.

Zeanah C, Boris N, Larrieu J. Infant development and developmental risk: A review of the past 10 years. Journal of American Child and Adolescent Psychiatry. 1997; 36:165-178.

Zuckerman B, Bauchner H, Parker S, Cabral H. Maternal depressive symptoms during pregnancy, and newborn irritability. Journal of Behavioral and Developmental Pediatrics. 1990; 11:190-194. 


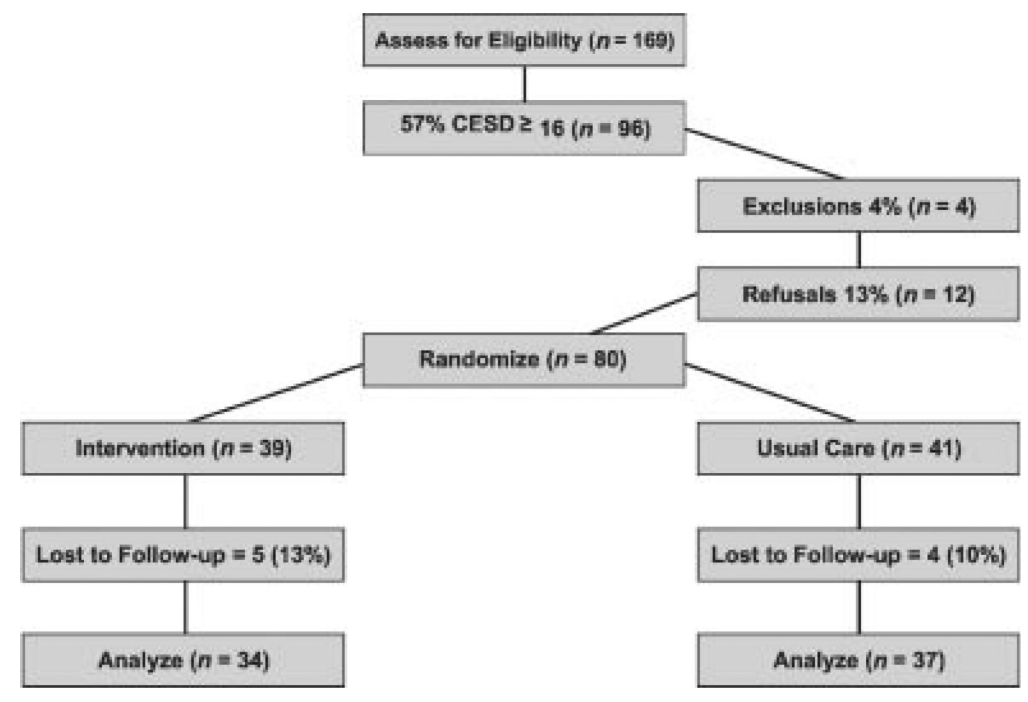

FIGURE 1.

CONSORT diagram of participant flow. 


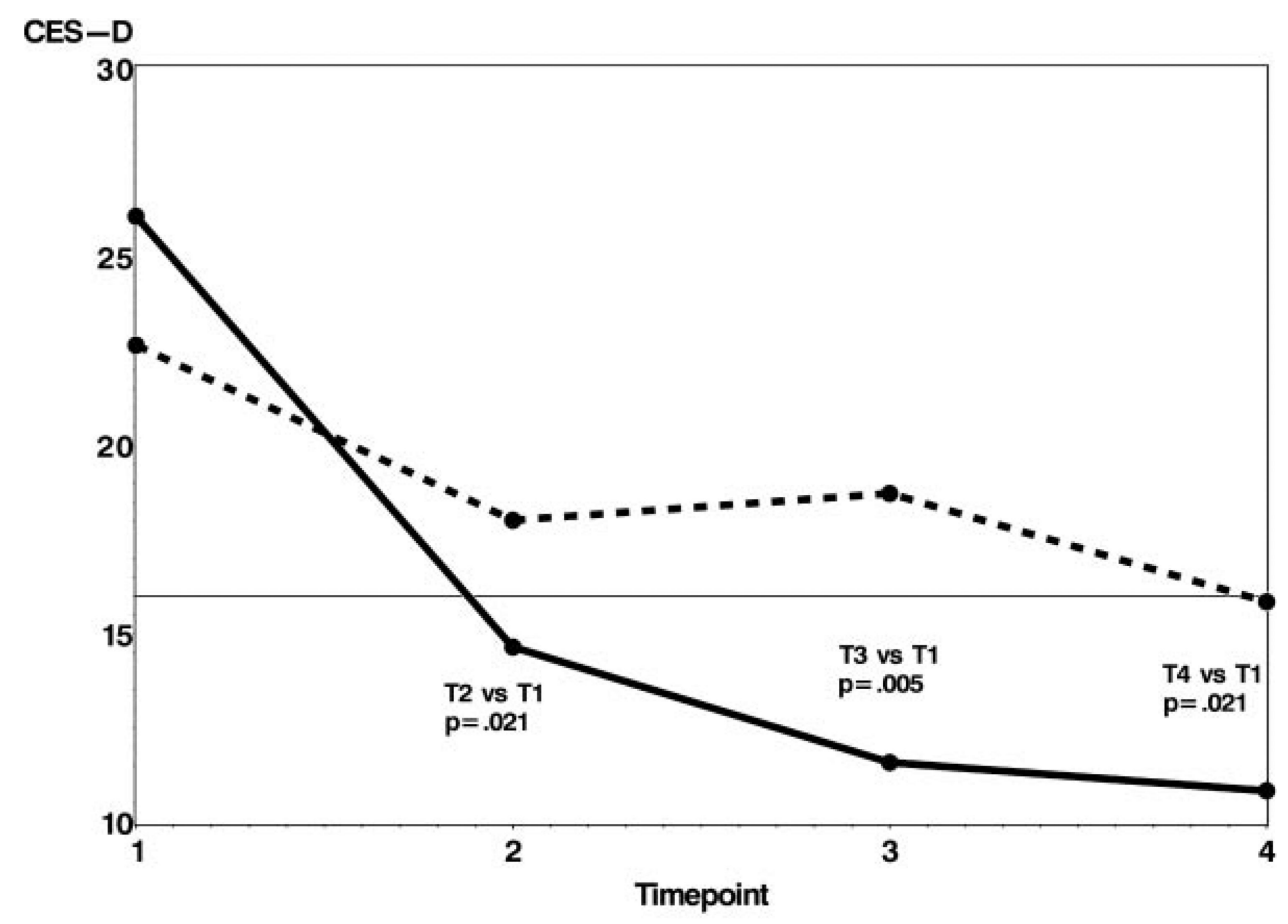

\section{Randomized Group \\ Control --.-- \\ Intervention}

FIGURE 2.

Within group changes in CES-D at each timepoint. 


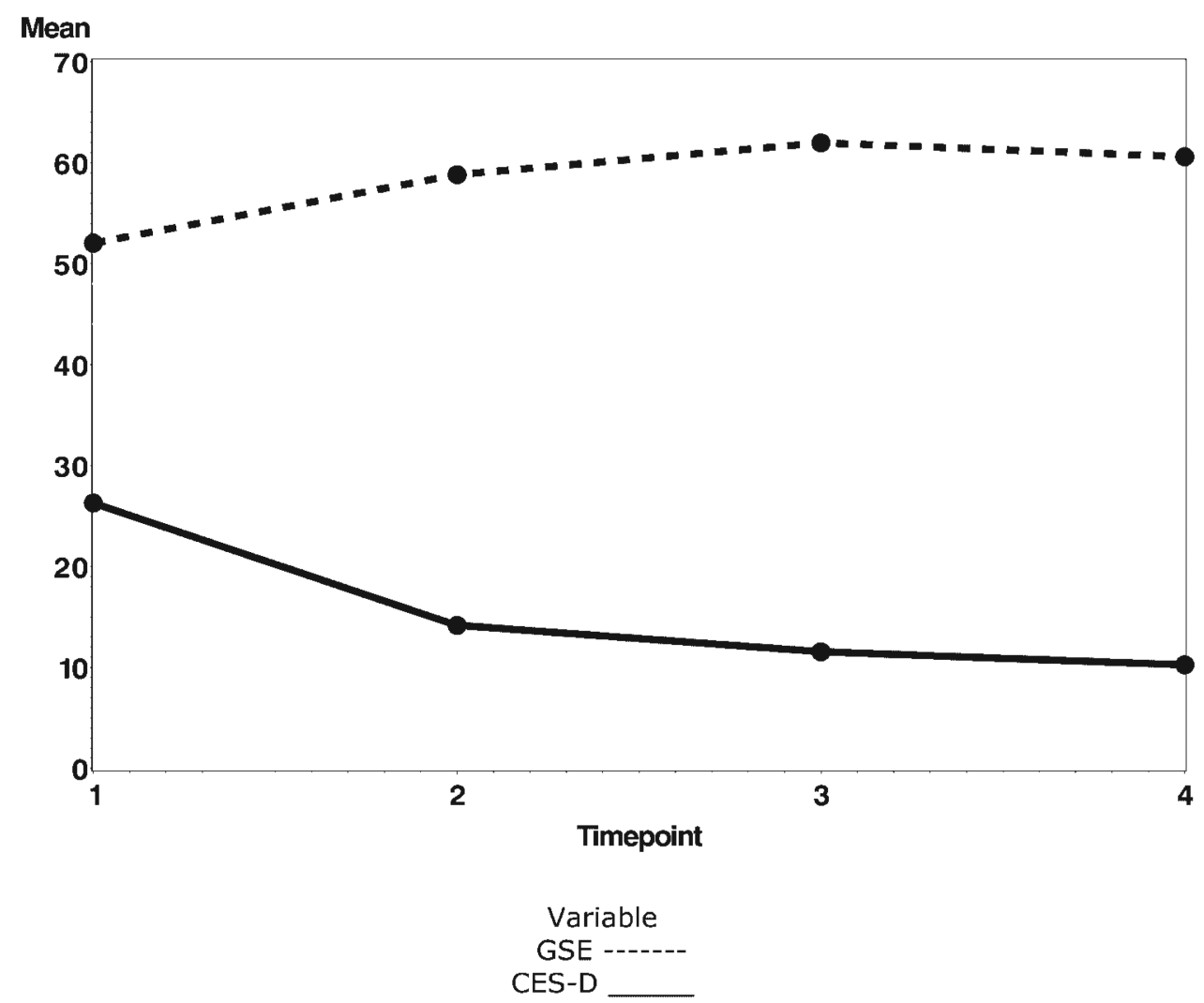

FIGURE 3.

Plot of intervention group GSE and CES-D change at each timepoint. 


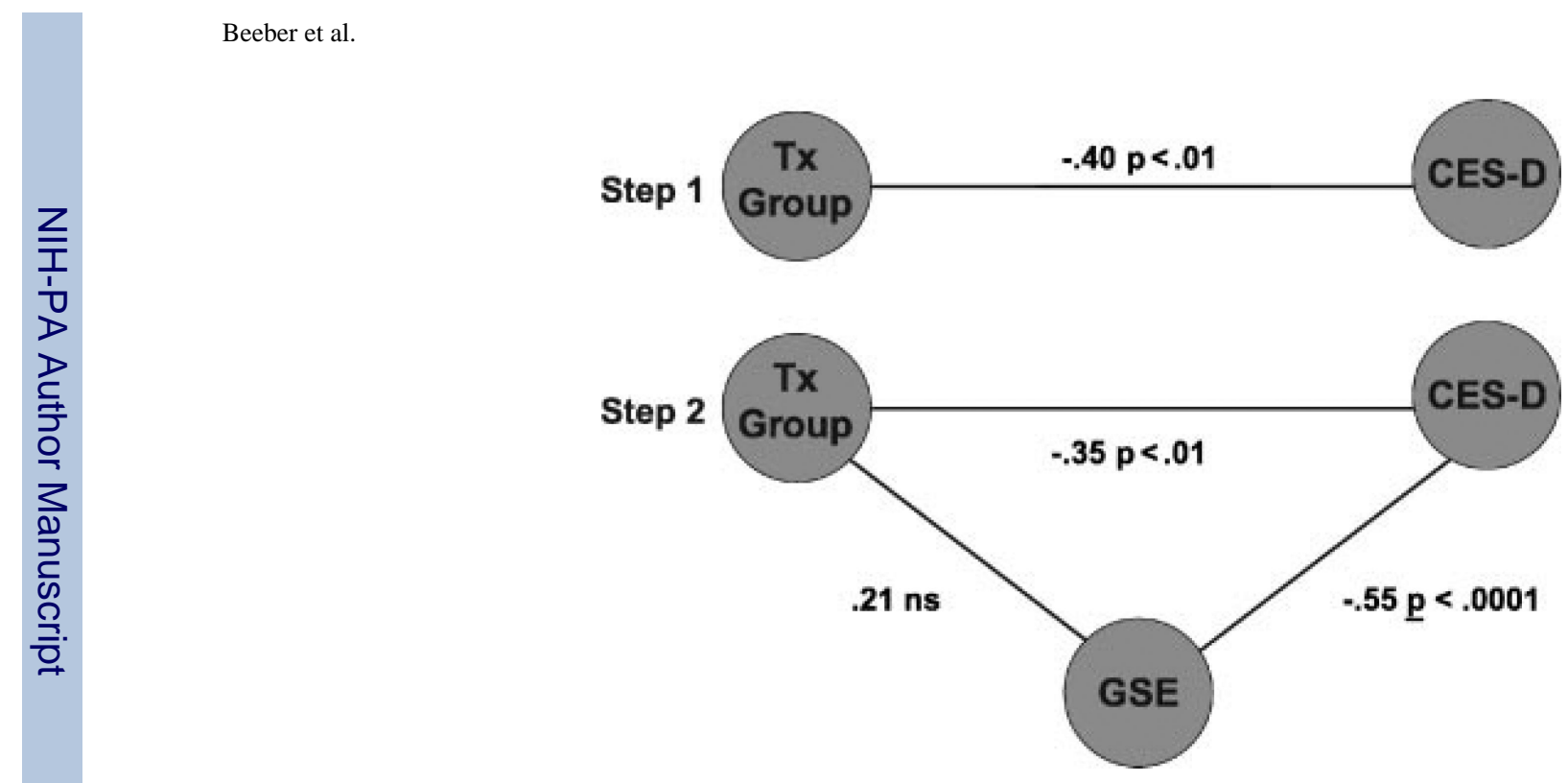

FIGURE 4.

Analysis of mediation effect of GSE in the intervention group. 


\section{Table 1}

\section{Demographic Characteristics}

\begin{tabular}{|c|c|}
\hline \multicolumn{2}{|l|}{ Maternal age } \\
\hline Intervention & $26.2(6.1)$ \\
\hline Usual care & $26.5(5.8)$ \\
\hline \multicolumn{2}{|c|}{ Years formal education } \\
\hline Intervention & $8.3(2.9)$ \\
\hline Usual care & $9.1(2.9)$ \\
\hline \multicolumn{2}{|c|}{ Working full-time or part-time } \\
\hline Intervention & $31 \%$ \\
\hline Usual care & $44 \%$ \\
\hline \multicolumn{2}{|c|}{ Living with partner } \\
\hline Intervention & $82 \%$ \\
\hline Usual care & $85 \%$ \\
\hline \multicolumn{2}{|c|}{ Annualized family income } \\
\hline Intervention & $\$ 15,809(\$ 6668)$ \\
\hline Usual care & $\$ 13,431(\$ 5399)$ \\
\hline \multicolumn{2}{|c|}{ Early head start child age in months } \\
\hline Intervention & $15.2(11.5)$ \\
\hline Usual care & $16.6(11.6)$ \\
\hline \multicolumn{2}{|c|}{ Early head start child gender $=$ female } \\
\hline Intervention & $54 \%$ \\
\hline Usual care & $51 \%$ \\
\hline
\end{tabular}


Table 2

Means and Standard Deviations on Key Variables by Group at Each Timepoint

\begin{tabular}{|c|c|c|c|c|}
\hline Construct/Measure & T1 & $\mathbf{T 2}$ & T3 & T4 \\
\hline \multicolumn{5}{|c|}{ Depressive symptom severity—CES-D } \\
\hline Intervention & $26.3(13.4)$ & $14.2(9.1)$ & $11.5(9.5)$ & $10.3(9.2)$ \\
\hline Usual care & $22.8(12.5)$ & $17.6(10.4)$ & $19.1(9.9)$ & $16.0(10.4)$ \\
\hline \multicolumn{5}{|c|}{ Maternal report of child aggression- $-\mathrm{CBCL}$, Aggression subscale raw score } \\
\hline Intervention & $16.7(10.8)$ & - & - & $11.2(8.1)$ \\
\hline Usual care & $16.8(7.5)$ & - & - & $15.4(9.4)$ \\
\hline \multicolumn{5}{|c|}{ Maternal concerns about child social-emotional competence-ASQ-SE } \\
\hline Intervention & $13.2(12.2)$ & - & - & $7.7(11.7)$ \\
\hline Usual Care & $15.0(12.0)$ & - & - & $9.4(12.7)$ \\
\hline \multicolumn{5}{|c|}{ Maternal responsiveness-HOME } \\
\hline Intervention & $33.2(7.2)$ & $33.6(5.4)$ & $35.4(5.8)$ & $36.0(6.5)$ \\
\hline Usual care & $33.0(6.4)$ & $34.5(6.3)$ & $36.2(5.5)$ & $36.3(6.2)$ \\
\hline \multicolumn{5}{|l|}{ Mediator mean (SD) } \\
\hline \multicolumn{5}{|c|}{ Maternal self-efficacy-GSE } \\
\hline Intervention & $52.0(12.5)$ & $58.8(10.4)$ & $62.0(12.1)$ & $60.6(11.7)$ \\
\hline Usual care & $55.3(12.4)$ & $62.6(10.9)$ & $60.1(10.8)$ & $60.8(11.8)$ \\
\hline \multicolumn{5}{|l|}{ Moderators mean $(S D)$} \\
\hline \multicolumn{5}{|l|}{ Acculturation } \\
\hline \multicolumn{5}{|l|}{ PAS } \\
\hline Intervention & $1.2(0.3)$ & $1.3(0.3)$ & $1.3(0.3)$ & $1.2(0.3)$ \\
\hline Usual care & $1.2(0.3)$ & $1.3(0.3)$ & $1.3(0.3)$ & $1.3(0.3)$ \\
\hline \multicolumn{5}{|l|}{ SASH } \\
\hline Intervention & $4.8(2.5)$ & $4.8(1.7)$ & $4.7(1.8)$ & $4.9(1.7)$ \\
\hline Usual care & $4.7(1.7)$ & $5.1(1.6)$ & $5.2(1.8)$ & $5.1(1.7)$ \\
\hline \multicolumn{5}{|c|}{ Maternal physical health-MOS SF-12 } \\
\hline Intervention & $40.2(6.1)$ & $43.2(6.1)$ & $46.4(5.5)$ & $45.7(5.4)$ \\
\hline Usual care & $40.6(5.7)$ & $41.4(7.4)$ & $43.4(6.4)$ & $43.5(6.0)$ \\
\hline
\end{tabular}

CES-D, Center for Epidemiological Studies Depression scale; CBCL, Child Behavior Checklist; ASQ-SE, Ages and Stages Questionnaire-SocialEmotional; HOME, Home Observation for Measurement of the Environment; GSE, General Self-Efficacy Scale; PAS, Psychological Acculturation Scale; SASH, Short Acculturation Scale for Hispanics; MOS SF-12 Medical Outcomes Study Short Form. 